\title{
TGFB1 (RS1800470 AND RS1800469) VARIANTS ARE INDEPENDENTLY ASSOCIATED WITH DISEASE ACTIVITY AND AUTOANTIBODIES IN RHEUMATOID ARTHRITIS PATIENTS
}

Tatiana Mayumi Veiga Iriyoda ${ }^{1, *}$, Tamires Flauzino², Neide Tomimura Costa², Marcell Alysson Batisti Lozovoy², Edna Maria Vissoci Reiche ${ }^{2}$, Andrea Name Colado Simão²

1.Pontifícia Universidade Católica do Paraná, Londrina (PR), Brazil; 2.Universidade Estadual de Londrina, Londrina (PR), Brazil.

*Corresponding author: tatimayumi54@gmail.com

\section{BACKGROUND}

The present study evaluated the association between TGFB1 + 869 T > C (rs1800470) and TGFB1 - 509 C > T (rs1800469) variants with susceptibility, disease activity, autoantibodies, and TGF- $\beta 1$ plasma levels in rheumatoid arthritis (RA) patients from Southern Brazilian population.

\section{METHODS}

A total of 262 patients with RA and 168 control individuals were tested for the TGFB1 variants using a TaqMan genotyping assay. Anti-cyclic citrullinated peptide (anti-CCP) antibody levels were determined by chemiluminescence immunoassay and rheumatoid factor (RF) by turbidimetric assay. The TGF- $\beta 1$ serum levels were measured by ELISA kit. Disease activity score in 28 joints (DAS28) classified RA patients into two groups of disease activity: remission/mild (DAS28 <3.2) and moderate/severe (DAS28 $\geq 3.2)$.

\section{RESULTS}

Patients and controls did not differ in sex, smoking and ethnicity. The median disease duration was ten years (4-9) and 135 $(53.1 \%)$ patients were in moderate/severe disease activity. Diminished TGF- $\beta 1$ levels were found in the RA group $(p<0.001)$. The TGFB1 + 869 T > C and -509 C > T variants, independently or in haplotype combination, were not associated with RA susceptibility. Regarding the TGFB1 + $869 \mathrm{~T}>\mathrm{C}$ variant, we did not find association between this variant and autoantibodies status, DAS28, erythrocyte sedimentation rate (ESR), C-reactive protein (CRP), and TGF- $\beta 1$ level in the overall RA group. Besides, patients with the TGFB1 - 509 TT genotype had a higher frequency of patients with DAS28 $\geq 3.2$ than those with the CC genotype $(\mathrm{OR}=2.58$ [1.04-6.42], $p=0.041$ ). Patients with RF or anti-CCP positive and TGFB1 + 869 CC genotype had lower TGF- $\beta 1$ plasma levels than those with the TT + TC genotype. Besides that, patients with high RF titles $(>90 \mathrm{U} / \mathrm{mL})$ and the TGFB1 $+869 \mathrm{TC}+\mathrm{CC}$ genotypes showed a high frequency of patients with DAS28 $\geq 3.2(p=0.037)$. However, no differences were observed between disease activity and TGFB1 869 genotypes in patients with high anti-CCP titles $(>15 \mathrm{U} / \mathrm{mL})$.

\section{CONCLUSION}

The TGFB1 + 869 T > C variant was associated with diminished TGF- $\beta 1$ plasma levels and moderate/severe activity disease only in seropositive RF patients. This is the first study showing that TGF- $\beta 1$ plasma levels can be modulated by the interaction between the TGFB1 +869 T > C variant and autoantibodies. However, the TGFB1 - 509 C > T variant was associated with moderate/severe activity disease, independently of autoantibodies positivity. Thus, our findings suggest that TGFB1 +869 T > C and $-509 \mathrm{C}>\mathrm{T}$ variants can predict activity disease in different RA patient subgroups.

\section{KEYWORDS}

Rheumatoid arthritis, Genetic variants, Transforming growth factor- $\beta 1$, rs1800470, rs1800469. 